\title{
DISEÑO A DOBLE CIEGO Y EL USO DE PLACEBOS PSICOLÓGICOS EN INVESTIGACIÓN DE RESULTADOS EN PSICOTERAPIA: ES POSIBLE? UN ESTUDIO PILOTO DE VIABILIDAD*
}

\section{DOUBLE BLIND DESIGN AND THE USE OF PSYCHOLOGICAL PLACEBOS IN OUTCOMES RESEARCH IN PSYCHOTHERAPY: IS IT POSSIBLE? A PILOT FEASIBILITY STUDY}

\author{
Eduardo H. Cazabat**
}

\begin{abstract}
*El estudio que se presenta forma parte de la Tesis Doctoral en preparación del autor. **Licenciado y Doctorando en Psicología, Master Traumatologist, Maestrando en Metodología de la Investigación Científica y Profesor Universitario de Psicología. Profesor Titular de Atención en Crisis y Catástrofes de la Universidad del Salvador (USAL) y de Seminario de Trauma y Disociación de la Universidad Católica de Santa Fe (UCSF).

E-Mail: eduardo.cazabat@yahoo.com.ar - http://www.psicotrauma.com.ar/cazabat Ángel J. Carranza 2400, Piso 12, Dpto. A - (C1425FXF) Ciudad Autónoma de Buenos Aires. República Argentina.
\end{abstract}

\section{Resumen}

El uso de placebos y diseños a doble ciego ha cumplido un papel crucial en la investigación clínica en Medicina. Su aplicación a la investigación de resultados en psicoterapia ha sido controversial. Muchos autores niegan la posibilidad de su aplicación debido a que el terapeuta debería conocer la condición del procedimiento aplicado. Se presenta detalladamente un estudio en el que se utilizó un procedimiento placebo correspondiente a EMDR (Eye Movement Desensitization and Reprocessing - Desensibilización y Reprocesamiento por Movimientos Oculares) con un Diseño Experimental de Caso Único. Los objetivos fueron los siguientes: poner a prueba la viabilidad de la implementación de un placebo de tipo psicológico en EMDR y la aplicación de un diseño a doble ciego en el estudio de resultados en EMDR.

Se seleccionaron tres pacientes que sufrían de Trastorno por Estrés Postraumático. Se estableció una línea de base de la sintomatología presentada. Luego, los sujetos fueron asignados aleatoriamente a tres condiciones experimentales durante tres sesiones: (a) aplicación del protocolo EMDR sin ningún tipo de estimulación con auriculares en silencio (placebo 1), (b) apli- cación del protocolo EMDR con estimulación bilateral auditiva simultánea (no alternada) (placebo 2) y (c) aplicación del protocolo estándar de EMDR con estimulación auditiva bilateral alternada (tratamiento activo).

Tales procedimientos resultaron igualmente creíbles para el paciente y para el terapeuta. Esto permitió el desarrollo de un diseño de investigación a doble ciego para la investigación de resultados en EMDR. Finalmente, se discuten algunas posibles aplicaciones e implicancias de la introducción del uso de placebos psicológicos y diseño a doble ciego en la investigación en psicoterapia.

Palabras clave: Investigación de resultados en psicoterapia; Doble ciego; Placebo psicológico; EMDR; Diseño experimental de caso único.

\section{ABSTRACT}

Double blind design and placebos have been of crucial importance in medical clinical research. Their use in outcomes research in the field of psychotherapy has been controversial, though. Their feasibility in such case has been denied by many authors based on the assumption that the psy- 
chotherapist would need to know the nature of the applied procedure. In view of this, the author has conducted a pilot feasibility study on three subjects within the context of his doctoral dissertation. Said dissertation aims at establishing the role of alternating bilateral auditory stimulation in the processing of traumatic memories as used in the EMDR (Eye Movement Desensitization and Reprocessing) technique. To such end, the EMDR basic principles and procedures are introduced -with particular attention to alternating bilateral auditory stimulation- and a pilot study using placebos during EMDR administration is presented in detail. The goals of this study are testing the feasibility of: (a) using a psychological placebo in EMDR therapy, and (b) applying a double blind design study in EMDR outcomes research.

A single case experimental design was performed on three different patients suffering from PTSD (Posttraumatic Stress Disorder). A symptomatology baseline was established throughout three weekly sessions using the DTS (Davidson Trauma Scale) and the OQ-45.2 (Outcomes Questionnaire 45.2). First, three CDs were recorded -one with no sound at all (CD-1, silence condition); another one with auditory stimulation consisting of a tic-tac sound recorded in monaural condition, and reproduced simultaneously over both earphones at a rate of one beat per second (CD-2, monaural condition) and a third one with alternating bilateral auditory stimulation consisting of the exact same sound recorded in stereophonic condition, and reproduced alternatively over the left and right earphones (CD-3, stereo condition)-. At a second stage, these three experimental conditions were assigned randomly to the three subjects, who were administered: (a) EMDR protocol without any type of stimulation, with no sound coming out of the earphones using CD-1 (placebo 1), (b) EMDR protocol with simultaneous bilateral auditory stimulation using CD-2 (placebo 2), and (c) EMDR protocol with alternating bilateral auditory stimulation using CD-3 (active treatment). In all cases, the experimental conditions were implemented during three full sessions in which the CDs were reproduced for the subjects through earphones, instead of speakers, to ensure that the psychotherapist was unaware of the actual conditions. Subsequently, the standard EMDR protocol (i.e., with alternating bilateral auditory stimulation) was administered to each subject until the end of the treatment, determined either by the symptoms being resolved or the maximum of ten sessions being completed.

As a result of this pilot study, the author concludes that the feasibility of using double blind studies and placebos in EMDR psychotherapy has indeed been established. Since no apparent difficulties in the administration of the placebos were detected during the study, the use of psychological placebos seems viable. Such procedure is equally credible for the patient as well as for the psychotherapist, which renders possible the development of a double blind design in EMDR outcomes research. It should be noted, though, that the credibility of the placebo was not formally assessed, but rather was perceived through the author's informal observation. Developing assessment criteria and formal tools to evaluate the credibility of placebo procedures is advisable if future investigations on the subject are to be carried. Even though this study was conducted under a Single Case Experimental Design, the placebo procedure employed could be easily adapted for its use in between group's designs. Finally, some of the possible applications and consequences regarding the introduction of placebos and double blind design in psychotherapy research are discussed.

Key words: Outcomes research in psychotherapy; Double blind; Psychological placebo; Single case experimental design; EMDR.

\section{INTRODUCCIÓN}

La introducción de la investigación a doble ciego en las Ciencias Médicas ha representado un hito importantísimo y dio el impulso para dejar atrás un largo período basado en supersticiones y creencias. La comprobación de la eficacia de los fármacos por medio de estudios controlados ha significado el ingreso de la terapéutica médica en el ámbito de la ciencia. Asimismo, la investigación desarrollada en el ámbito de la psicoterapia y sus resultados ha sido desarrollada a partir de 1952, luego de que Eysenck publicara The ef- 
fects of psychotherapy: An evaluation (Carr, 2009; Freeman \& Power, 2007; Rubin, 2008; Santibáñez Fernández, Román Mella \& Vinet, 2009) y ha ido perfeccionándose metodológicamente a lo largo de las últimas décadas con la introducción de los diseños experimentales y la estadística.

Sin embargo, aún falta que las psicoterapias prueben su efectividad mediante el empleo de diseños a doble ciego. Muchos autores han negado la posibilidad de realizar estudios a doble ciego en psicoterapia, dado que, según argumentan, el psicoterapeuta que aplica el tratamiento, no podría desconocer la naturaleza del tratamiento que está aplicando. No obstante, aunque implícitamente, ha sido aceptada esta posibilidad por algunos pocos autores (Chambless et al., 1996; Herbert, 2003; Herbert \& Gaudiano, 2005).

En este artículo se abordará el tema, planteando una forma posible de realizar este tipo de estudios, al menos en algunas psicoterapias. A fin de determinar la factibilidad de la utilización de un placebo de tipo psicológico y la factibilidad de utilizar un diseño a doble ciego en el estudio de resultados en psicoterapia, se realizó un estudio piloto con tres casos. Para ello se utilizó un Diseño Experimental de Caso Único, estableciendo la factibilidad de la realización de tal tipo de estudios. Finalmente, se plantean algunas posibles líneas de investigación que se pueden derivar de este tipo de estudios controlados a doble ciego con placebo en psicoterapia.

\section{EL USO de dROGAS EN LOS ORÍGenes DE LA TERA- PÉUTICA MÉDICA}

En sus orígenes, la terapéutica médica se basaba en supersticiones y creencias, usando drogas muchas veces inocuas cuando no perniciosas, y adoptando en muchos casos formas bizarras (Wojciechowski, 1984). Hasta años recientes, las terapéuticas médicas sólo funcionaban basadas en la fe, la esperanza o la relación médico-paciente. El paradigma detrás de la Medicina se basaba en la falacia post hoc, ergo propter hoc (si B ocurre con posterioridad a A, A es la causa de B). Se ha dicho que la historia de la Medicina es la historia del efecto placebo, dado que la inmensa mayoría de las terapéuticas aplicadas carecían de principios activos y se basaban, como queda dicho, en la superstición, la fe, la esperanza, etc. tanto del paciente como del médico (Lilienfeld, Ritschel, Lynn, Cautin \& Latzman, 2013; Wojciechowski, 1984).

\section{LOS ESTUDIOS DOBLE CIEGO EN LOS ESTUDIOS CLíNICOS EN MEDICINA. ENSAYOS CLínICOS CONTRO- LADOS}

Los primeros estudios pioneros en este campo fueron aislados. En 1747, Lindt diseñó un ensayo controlado comparando seis terapéuticas diferentes en sendos grupos de pacientes que sufrían de escorbuto, demostrando que los únicos que mejoraron fueron los que recibieron cítricos como tratamiento. En 1793, Cobett aplicó las estadísticas por primera vez para demostrar que la terapéutica usual por entonces para tratar la fiebre amarilla, consistente en sangrías y purgas, no sólo no era efectiva, sino que provocaba daño a los pacientes. Louis, contemporáneamente, planteó la necesidad de comparar el efecto de diferentes tratamientos, y al mismo tiempo en la Sociedad Médica de Viena se llevaban a cabo estudios en los que tanto los pacientes como los médicos desconocían la medicación usada (Wojciechowski, 1984).

Los avances de la Química Orgánica en el Siglo XIX junto con los métodos de investigación desarrollados, proveyeron a la $\mathrm{Me}-$ dicina las herramientas necesarias para el nacimiento de la investigación clínica objetiva. En la década de 1930 se formalizaron las principales características del modelo de investigación clínica cuyos componentes principales, según Wojciechowski (1984) son:

a.- una población objeto de estudio claramente definida,

b.- la asignación aleatoria a los diversos tratamientos de las variables independientes,

c.- métodos estandarizados de medida de los resultados, 
d.- la incorporación de las estadísticas y e.- la comparación con placebos o con grupos control sin tratamiento.

El principio del uso de grupos de control ha sido conocido por más de 2.000 años. En cambio, el principio de aleatorización fue formulado recién hacia fines del Siglo XIX (Krauth, 2000).

\section{LA INVESTIGACIÓN DE RESULTADOS EN PSICOTERAPIA}

Según Lambert y Goates (2002) los estudios sobre la eficacia de la psicoterapia ponen el acento en la validez interna del diseño experimental, con el objetivo de establecer claramente la relación entre el tratamiento y el resultado. Este tipo de estudios se caracteriza por la asignación aleatoria de los pacientes a los tratamientos, la administración de tratamientos estandarizados por medio de su manualización, la dosificación del tratamiento, el entrenamiento y monitorización de los terapeutas y la selección minuciosa de pacientes, entre otras medidas para garantizar el control experimental. Por otra parte, los estudios sobre efectividad están relacionados con los ámbitos naturales donde se aplican las psicoterapias, poniendo el acento en la validez externa, en un intento de demostrar que el tratamiento tiene efectos benéficos en el marco de un ámbito clínico típico. Según los autores, ambos términos frecuentemente se han usado indistintamente en la literatura, generando confusión.

Los primeros estudios sobre los resultados de la psicoterapia fueron llevados a cabo por Eysenck y publicados en 1952 bajo el título The effects of psychotherapy: An evaluation, y se centraron en las psicoterapias psicodinámicas (Carr, 2009; Cazabat, 2013; Freeman \& Power, 2007; Rubin, 2008).

En 1966, considerado el año en que se inicia la investigación experimental en psicoterapia, Gordon Paul planteó que la investigación debería determinar qué tipo de tratamiento, para qué tipo de consultante y en qué circunstancias sería más efectivo, y cuál sería la causa de esa efectividad. Y que, en este marco, la determinación de la efectividad debería establecerse en comparación con un placebo (Chambless et al., 1998; Chambless \& Ollendick, 2001; Herbert \& Gaudiano, 2005). Paul (1966, citado en Herbert \& Gaudiano, 2005) definió el placebo como

"un tratamiento en el cual los sujetos tienen igual confianza, pero que no se esperaría que conduzca a un cambio conductual en ningún terreno" (p. 898).

En la misma dirección, la American Psychological Association recogió este criterio al desarrollar listas de tratamientos con apoyo empírico (Borkovec \& Sibrava, 2005; Chambless et al., 1998; Chambless \& Ollendick, 2001; Herbert \& Gaudiano, 2005). De tal manera que en los criterios para considerar el apoyo empírico de un tratamiento se requieren al menos dos pruebas en las que el tratamiento experimental sea superior (estadísticamente significativo) a un placebo psicofarmacológico o psicológico, o a otro tratamiento (Chambless et al., 1998).

\section{EL uso de PLACEBOS en INVESTIGACIÓN}

El uso de placebos y/o de grupos de control sin tratamiento tiene por objeto determinar los efectos del paso del tiempo, la remisión espontánea o el efecto de estar en tratamiento, aislando de esta manera los efectos específicos de la medicación (Herbert \& Gaudiano, 2005; Wojciechowski, 1984). Para que el estudio cumpla sus objetivos, el paciente no debe saber si está recibiendo el tratamiento con el principio activo o el placebo, para no influir en sus respuestas. Aún más, sería deseable que no supiera siquiera que participa en un estudio hasta la finalización del mismo (Heppner, Wampold \& Kivlighan Jr., 2008; Howitt \& Cramer, 2011). Esta condición es la de simple ciego, es decir, el paciente es ciego al tipo de tratamiento recibido. Desde el punto de vista tradicional de la investigación en psicoterapia, éste sería el mayor estándar alcanza- 
ble: que el paciente no supiera si está recibiendo el tratamiento activo o no, dado que el terapeuta estaría obligado a saber si provee uno u otro, dadas las características de los tratamientos psicoterapéuticos tradicionales. De esta manera se previenen los efectos de las expectativas del paciente. Habitualmente esto se logra por medio de listas de espera (el paciente no recibe ningún tratamiento), o recibe sesiones inespecíficas de apoyo, en el intento de igualar la atención recibida con la de aquel que recibe el tratamiento activo.

\section{¿Qué Es Un ESTUdio a dOBLE CIEgo?}

Pero no sólo el paciente tiene expectativas sobre el tratamiento. El clínico (o el investigador) también las tiene, e influyen en los resultados (efecto Rosenthal) (Heppner et al., 2008). Es así que, en la década de 1940, se desarrolla el diseño de estudio a doble ciego. De esta manera, siendo el clínico/investigador ciego al tratamiento que administra (activo o placebo) se controla el efecto de las expectativas del mismo sobre los resultados del estudio. Por lo tanto, el diseño a doble ciego controla el posible sesgo que surja tanto de parte del paciente como del experimentador. De hecho, en algunos casos, la comparación de resultados de estudios del efecto de ciertas drogas que a simple ciego demostraron ser efectivas, en estudios a doble ciego demostraron no ser más efectivas que el placebo (Wojciechowski, 1984).

Este procedimiento es fácilmente imaginable en el ámbito de la Medicina Clínica: se administra a algunos pacientes una medicación activa, mientras que a otros se les administra el placebo (un medicamento del mismo aspecto pero sin principio activo), sin que el médico o investigador sepa cuál de los dos se le está administrando. Sin embargo, es más difícilmente adaptable al ámbito de la psicoterapia, aunque como se verá más adelante, es posible, al menos en ciertos casos.

La condición de triple ciego es aquella en la que el evaluador es una persona diferente del terapeuta / investigador, y también des- conoce si está evaluando a un participante tratado con placebo o tratamiento activo.

\section{EL USO DE PLACEBOS EN LA INVESTIGACIÓN EN PSI- COTERAPIA}

El uso de placebos en la investigación en psicoterapia es un tema controversial.

Borkovec y Sibrava $(2005$, p. 805) afirman que

"debido a numerosos problemas de orden conceptual, metodológico y ético que están asociados con el uso de placebo en investigación en psicoterapia, su uso debería ser abandonado...”.

Los problemas mencionados por los autores son tres:

a.- El primero es de orden conceptual: si se administra un tratamiento inerte y aun así ocurre algún cambio, éste se debería a algún tipo de mecanismo psicológico, por lo cual el placebo no era inerte,

"por lo tanto, metodológicamente, es imposible crear un procedimiento psicológico de control que sea inerte" (p. 807).

b.- Los autores asumen que el terapeuta / investigador necesariamente estará al tanto de estar administrando un tratamiento placebo, contaminando con sus expectativas los resultados. Asimismo afirman que es prácticamente imposible crear un procedimiento placebo con el mismo grado de credibilidad que el tratamiento activo.

c.- Finalmente, plantean el problema ético de mantener en un tratamiento inerte a un paciente por un plazo determinado, que podría ocasionar un daño.

Otros autores que se pronuncian negativamente acerca de la posibilidad del uso de placebos y de diseño a doble ciego en psicoterapias son los siguientes:

1.- Herbert y Gaudiano (2005, p. 896): “a diferencia de las pruebas con drogas, la psi- 
coterapia tradicional no puede ser administrada a ciegas". Sin embargo, estos autores reconocen la posibilidad de realizarlo en las "así llamadas power therapies" (p. 896).

2.- Borkovec y Sibrava (2005, p. 807): "es casi imposible desarrollar una condición de placebo psicológico real adecuado."

3.- Seligman (1995, citado en Barker, Pistrang \& Elliot, 2002, p. 158): "sin embargo, usualmente no es posible realizar estudios a doble ciego o triple ciego en psicología, tal como se realiza en medicina, desde el momento en que el terapeuta sabe qué tratamiento está dando, y los pacientes saben, normalmente qué están recibiendo."

4.- Haworth (1996, p. 2): "Este procedimiento doble ciego, que es usado en pruebas clínicas de ciertas drogas, es obviamente muy difícil, si no imposible de emprender con ciertas formas de psicoterapia."

5.- Barkham, Hardy y Mellor-Clark (2010, p. 19): "En estudios sobre terapias psicológicas, los diseños a doble ciego son totalmente imposibles, y los estudios simple ciego son virtualmente imposibles. El psicoterapeuta debe conocer qué tratamiento se aplica y, desde el punto de vista ético, el paciente debe conocer los tipos de intervención a los que puede haber sido sometido en forma aleatoria."

6.- Marczyk, DeMatteo y Festinger (2005, p. 75): "Por una variedad de razones, frecuentemente no es práctico o apropiado usar un procedimiento doble ciego."

7.- Simons y Wildes (2005, p. 339): “¿Pero cómo un terapeuta puede hacer esto si conoce que el tratamiento fue diseñado para ser inerte? Éste y otros problemas han llevado a reclamar el abandono de las comparaciones con placebo en la investigación en psicoterapia y la búsqueda de diseños alternativos para abordar la cuestión de la especificidad de los efectos (DeMarco, 1998; Seligman, 1995; Parloff, 1986; Wampold, 2001)".
8.- McLeod (2003, p. 123): "En investigación sobre drogas (pero no en investigación en psicoterapia o counselling) es posible realizar estudios a doble ciego."

9.- Roberts e Ilardi (2005) y Goodwin (2010) hacen referencia únicamente al doble ciego y el uso del placebo en investigación en farmacoterapia.

10.- Borkovec y Onken (2002) sintetizan todos estos argumentos de la siguiente manera:

"Conceptualmente, no es posible diseñar placebos psicológicamente inertes. (...) Metodológicamente, la evidencia empírica indica que, porque frecuentemente no son creíbles para los clientes, los placebos psicológicos no evocan el mismo nivel de expectativa de mejora que las intervenciones conductuales. También es imposible mantener al terapeuta (y frecuentemente al cliente) ciego a si el cliente está recibiendo el placebo o el tratamiento activo. Éticamente, a causa de que las intervenciones conductuales a menudo se desarrollan a lo largo de largos períodos, (por ej. en la investigación típica sobre resultados de la psicoterapia), es difícil para los terapeutas continuar proveyendo una intervención que se cree que es considerablemente menos efectiva que la comparada en la condición experimental" (p. 307).

Por otra parte, sendas búsquedas realizadas en las bases EBSCO, PILOTS y MEDLINE, con las palabras clave psychotherapy y double blind research sólo arrojaron resultados relacionados con investigaciones con fármacos, y ninguno exclusivamente con psicoterapia, excepción hecha de la recensión del libro de Wojciechowski (1984).

\section{¿QUÉ ES UN PLACEBO PSICOLÓGICO?}

Según Shapiro y Morris (1978, citado en Borkovec \& Sibrava, 2005), placebo es 
"cualquier terapia o componente de terapia que es usado deliberadamente por su efecto psicológico o psicofisiológico no específico, o que es usado por su efecto presupuesto, pero que no tiene actividad específica para la condición que está siendo tratada" (p. 805).

En el ámbito específico de la psicoterapia, un placebo debería

"controlar los factores terapéuticos (por ej. expectativas, relación terapéutica, esperanza, credibilidad del tratamiento) excepto las técnicas o procesos (por ej. exposición a estímulos temidos, trabajo de la transferencia) que según se cree o teoriza son los que producen el efecto del tratamiento sometido a estudio" (Herbert \& Gaudiano, 2005, p. 895).

Si bien es difícil de imaginar que un psicoterapeuta de orientación psicoanalítica, gestáltica, sistémica, cognitiva, entre otras, podría ignorar si está aplicando un principio activo de la psicoterapia que está desarrollando, en cambio hay otros que se verán ahora, en que esto sí es posible.

La psicoterapia conocida como EMDR (Eye Movement Desensitization and Reprocesing - Desensibilización y Reprocesamiento por Movimientos Oculares) desarrollada por Shapiro $(1995,2001)$, en algunas de sus formas de aplicación, es pasible de ser administrada sin que el terapeuta esté al tanto de qué tipo de estimulación se está administrando, como se verá a continuación.

\section{HISTORIA DEL EMDR}

El EMDR fue desarrollado por Shapiro, quien en 1987 descubrió accidentalmente que, mientras estaba concentrada en pensamientos perturbadores, al realizar cierto movimiento rápido e involuntario de ojos, la perturbación asociada al pensamiento se diluyó, desapareciendo totalmente (Shapiro, 1995). Éste fue el comienzo de un proceso de investigación, primeramente informal, y que luego llegó a involucrar grupos de veteranos de Vietnam y de mujeres violadas (Oren \& Solomon, 2012; Shapiro, 2007), en los que dicha técnica fue demostrando su eficacia.

Desde aquel comienzo como una simple técnica, EMDR se ha desarrollado hasta constituir en el presente un abordaje psicoterapéutico integrador (Shapiro, 2007).

En sus inicios fue denominado Eye Movement Desensitization (EMD) y se lo conceptualizó como un procedimiento de desensibilización, en la tradición de la terapia de conducta (Oren \& Solomon, 2012; Shapiro, 2007). Con el pasar del tiempo, se descubrió que no sólo los movimientos oculares podían provocar el procesamiento, sino también que otras formes de estimulación podían desencadenar similares procesos (Shapiro, 2007). Asimismo, también se fue haciendo evidente que algo más que la mera desensibilización ocurría, detectándose la aparición de insights, cambios espontáneos en conductas, emociones y sensaciones, de tal manera que el trauma se transformaba en nuevos aprendizajes. Esto determinó, en 1991, el cambio de nombre al actual de $E M D R$, al añadir el reprocesamiento (Oren \& Solomon, 2012; Shapiro, 2007).

Este concepto de reprocesamiento de las experiencias archivadas neurobiológicamente, por medio de un proceso de aprendizaje rápido, se convirtió en el eje teórico que guía el EMDR, y que se plasma en la formulación del modelo de Procesamiento Adaptativo de la Información (Shapiro, 2007).

Si bien en sus orígenes fue desarrollado y aplicado específicamente a pacientes que presentaban síntomas de índole postraumática, su espectro de aplicación no se reduce exclusivamente a tal tipo de pacientes. Se aplica actualmente con éxito en trastornos y síntomas tales como fobias, dolor, ansiedad, disociación, situaciones críticas y de desastre, etc. (Maxfield, 2007; Shapiro, 2001, 2012).

\section{EMDR: SUPUESTOS BÁSICOS}

El primer presupuesto del que se parte en el EMDR, es que existe en el ser humano un 
mecanismo natural de curación de las secuelas de los eventos traumáticos (Shapiro, 2001; Spector, 2007). Dado que todos sufrimos a lo largo de la vida innumerable cantidad de situaciones potencialmente traumatogénicas, y sólo una pequeña proporción de ellas provocan alguna consecuencia, es de suponer que algún mecanismo interno, innato, no aprendido, hace que esa información sea archivada de manera que no perturbe al individuo (Rothbaum \& Davis, 2004; Shapiro, 2001; Spector, 2007).

La información de estos traumas quedaría pues archivada en el sistema nervioso, de una manera específica, como una memoria dependiente de estado, de manera tal que continuarían influyendo en la conducta y la personalidad del individuo (Shapiro, 2001; Spector, 2007). De acuerdo con el Modelo de Procesamiento Adaptativo de la Información, formulado como hipótesis de trabajo por Shapiro, los recuerdos son archivados en redes neuronales interconectadas entre sí (Shapiro, 2001; Spector, 2007).

En el caso de los eventos traumáticos, la intensa reacción fisiológica de estrés y terror, bloquearía el procesamiento de la información recibida en el momento del trauma, la cual quedaría archivada bajo la forma de sensaciones, percepciones, recuerdos, creencias e ideas perturbadoras, de la misma manera en que fueron experimentadas originalmente. El procesamiento de esta información queda bloqueado, y diversos estímulos externos e internos asociados al trauma pueden disparar las perturbaciones bajo la forma de flashbacks, re-experimentaciones, pesadillas, activación fisiológica, etc. La red neuronal en la que esta información está depositada parece estar aislada de la información adaptativa contenida en otras redes (Shapiro, 2001; Spector, 2007). Es así, que muchos de los consultantes que sufren algún tipo de patología postraumática (desde una fobia simple a un estrés postraumático severo) saben que no deberían sentirse así (amedrentado por un simple gato o reaccionar como si estuviera bajo fuego enemigo al escuchar un portazo), pero no pueden reaccionar de otra manera.

\section{EL PROCEDIMIENTO DEL EMDR}

La estimulación provocada por el procedimiento del EMDR parece poner en funcionamiento un mecanismo por el cual la información perturbadora proveniente del trauma se pone en contacto con información adaptativa ('el gato no es un león' o 'el portazo no es fuego enemigo'), produciendo así un aprendizaje, muchas veces acompañado de un cambio cognitivo (Shapiro, 2001; Spector, 2007).

En la fase denominada desensibilización del tratamiento EMDR, se pone en marcha el procedimiento de estimulación bilateral alternada que desencadenará el procesamiento adaptativo (Shapiro, 2001; Spector, 2007). Para ello, se pide al paciente que focalice su atención en la situación elegida como blanco de la estimulación (el recuerdo traumático) y en las imágenes, cogniciones, sentimientos y sensaciones físicas asociados. Una vez hecho esto, el clínico comienza la estimulación bilateral alternada. Esta puede consistir en movimientos de ojos, para lo cual el terapeuta puede pedir que el paciente siga sus dedos mientras describe un camino de ida y vuelta hacia ambos extremos del campo visual del mismo. La estimulación puede ser también de orden kinestésico, golpeando alternada y suavemente en la palma de ambas manos o en las rodillas del paciente. También puede ser auditiva, por medio de sonidos alternantes en ambos oídos (existen grabaciones de sonidos o músicas especialmente preparadas para que el paciente los escuche por medio de auriculares). Esta estimulación bilateral alternada iría provocando el reproce samiento de la información asociada al hecho traumático, de tal manera que pueden cambiar las emociones, las sensaciones, las cogniciones, etc. Al cabo de cada tanda de estimulación el clínico pide que vuelva a concentrarse y pregunta "¿qué es lo que surge ahora?". No hay nada predeterminado en cuanto a lo que debería obtenerse. Mientras el material que el paciente produzca tenga alguna carga negativa, se prosigue con la estimulación bilateral alternada, haciendo blanco en este material, hasta que se llegue a un material no cargado 
negativamente (neutro o positivo). En ese momento se pide al paciente que vuelva a la situación original y se vuelve a evaluar su reacción. Mientras la reacción sea negativa, se continúa la estimulación bilateral alternada. La fase se concluye cuando al concentrarse el paciente en el blanco original, su reacción es neutra o positiva (Shapiro, 2001; Spector, 2007).

\section{ESTIMULACIÓN BILATERAL ALTERNADA}

Uno de los temas que plantea esta nueva terapia es el del papel que desempeña la estimulación bilateral alternada en el procesamiento de las memorias traumáticas.

Algunos autores afirman que los movimientos oculares no juegan ningún papel activo, y que EMDR es una variante de la Terapia de Exposición (Albright, Thyer, Becker, \& Rubin, 2011; Cusack \& Spates, 1999; Davidson \& Parker, 2001; Devilly, 2005; Herbert \& Sageman, 2004; McNally, 1999). Refiriéndose a EMDR, dice McNally (1999, citado en Nowill, 2010, p. 619):

“... no ofrece un tratamiento nuevo, sino un inteligente marketing, un nuevo embalaje de otros tratamientos validados de TEPT (por ej. Terapia de Exposición) o un placebo, y los Movimientos Oculares son una maniobra distractora: (...) lo que es efectivo en EMDR no es nuevo, y lo que es nuevo no es efectivo".

El mismo McNally (1999) afirma que Shapiro creó EMDR a partir de la Psicoterapia por Inhibición Recíproca de Wolpe, reemplazando la relajación muscular progresiva por los movimientos oculares en la función de inhibidor recíproco. Curiosamente, Shapiro informa haber recibido un apoyo inicial importante de parte de Wolpe cuando éste publicó en 1991 un reporte muy favorable a EMDR (Shapiro, 2012; Spector, 2007).

Por otra parte, los autores que apoyan el EMDR proponen que la estimulación bilateral alternada facilita la integración de la in- formación a nivel neurológico (ServanSchreiber, Schooler, Dew, Carter \& Bartone, 2006). Otros autores (Propper \& Christman, 2008) hipotetizan que la eficacia de EMDR está relacionada con el efecto de la estimulación sobre las estructuras neurofisiológicas de la memoria, al favorecer la comunicación interhemisférica.

Este tema ha suscitado un gran debate, acerca de si la estimulación bilateral alternada constituye un ingrediente activo del tratamiento. Si se demostrara que tal estimulación no cumple algún papel terapéutico, el EMDR quedaría reducido a una variante de la Terapia de Exposición (Bergman, 2000; Lee, Taylor \& Drummond, 2006; Spector, 2007). Algunas de las investigaciones realizadas hasta el momento postulan diversos efectos del movimiento ocular en las memorias visuales (Stickgold, 2002; Christman, Garvey, Propper \& Phaneuf, 2003). La mayoría de los estudios realizados en este terreno fueron realizados con estimulación por medio de movimientos oculares (Rubin, 2003). Otras formas de estimulación bilateral alternada, tal como la auditiva, no han recibido similar atención de los investigadores. De todas maneras, resulta claro que aún no está inequívocamente establecido el papel de la estimulación bilateral alternada en el EMDR, requiriéndose más investigación en los mecanismos de cambio, tanto de los específicos como de los inespecíficos (Ehlers et al., 2010; Lee et al., 2006; Maxfield, 2007; Schubert \& Lee, 2009).

En el estudio piloto realizado se exploró la factibilidad de utilizar la Estimulación Auditiva Bilateral Alternada en relación a la posibilidad de: (a) el uso de placebos y (b) la realización de estudios a doble ciego, en esta forma de psicoterapia.

\section{EL ESTUDIO PILOTO DE VIABILIDAD}

Los objetivos de este estudio fueron los siguientes: (1) poner a prueba la viabilidad de la implementación de un placebo de tipo psicológico en la psicoterapia denominada $E M D R$ y (2) poner a prueba la viabilidad de 
la aplicación de un diseño a doble ciego en el estudio de resultados en dicha psicoterapia.

\section{MÉTOdO \\ MAteRIALES}

Primeramente se prepararon tres discos compactos (CD) grabados de la siguiente manera: uno de ellos con la estimulación bilateral alternada propia del protocolo EMDR, consistente en un sonido de tipo tic-tac que se escucha alternadamente por cada auricular a una frecuencia aproximada de un sonido por segundo. Un segundo disco compacto con el mismo sonido grabado en condición monoaural, es decir que el sonido es emitido no alternadamente, sino simultáneamente por ambos auriculares. Y un tercer disco compacto con una grabación en silencio absoluto. Para su reproducción se utilizó un reproductor de discos compactos con auriculares personales estereofónicos.

Para monitorear la evolución del tratamiento, se administró la DTS (Davidson Trauma Scale - Escala de Trauma de Davidson) (Davidson et al., 1997; Villafañe, Milanesio, Marcellino \& Amodei, 2003) que evalúa la frecuencia y severidad de la sintomatología postraumática. Para evaluar el funcionamiento global, también se administró el OQ-45.2 (Outcomes Questionnaire 45.2 Cuestionario de Resultados 45.2) (Fernández Álvarez, Hirsch, Maristany \& Torrente 2005; Lambert, Hansen \& Bauer, 2008; Maristany \& Fernández Álvarez, en prensa; von Bergen $\&$ de la Parra, 2002).

\section{Procedimiento}

Los discos compactos fueron rotulados aleatoriamente con las letras A, B, y C y cada uno de ellos fue asignado aleatoriamente a los pacientes que participaron voluntariamente del estudio.

Los participantes en este estudio fueron reclutados por medio de anuncios verbales boca a boca y por medio de foros de correo electrónico, realizados entre colegas psico- terapeutas y alumnos universitarios de la Carrera de Psicología, ofreciendo un tratamiento gratuito de hasta 10 sesiones a quien sufriera las consecuencias de un evento traumático que se pudiera evaluar con la DTS. Por lo tanto, los participantes forman parte de una población clínica, aunque debe destacarse que la consulta no se dio espontáneamente.

Los sujetos firmaron un consentimiento informado y se les explicó que el estudio consistía en una comparación de los efectos de diversos tipos de estimulación en el tratamiento de los recuerdos traumáticos. También se les explicó que podían escuchar tanto un sonido alternante, como uno simultáneo, como un sonido subliminal (que no escucharían). Y se les pidió que no hicieran comentarios al terapeuta acerca del tipo de estimulación recibida. Si algún participante revelara algún detalle de la estimulación recibida (hecho que no sucedió), los tres participantes serían apartados del estudio, debiéndose comenzar el proceso desde el inicio, dado que de esta manera se suministrarían pistas para poder deducir o intuir el tipo de estimulación recibida por los otros dos participantes en el estudio. Finalmente se les explicó que en el curso del tratamiento podría variar el tipo de estimulación suministrada. Una vez finalizada la fase experimental, al comienzo de la cuarta sesión se suministró Estimulación Auditiva Bilateral Alternada, propia del protocolo de EMDR, por medio de otro disco compacto identificado previamente como tratamiento activo, hasta la finalización del tratamiento, ya sea por haber agotado las 10 sesiones o por la resolución sintomática.

En cada sesión se administró el OQ-45.2 para ver la evolución del tratamiento y la DTS para evaluar la evolución de la sintomatología postraumática en particular.

\section{DISEÑO DEL ESTUDIO}

Para este estudio piloto se aplicó un diseño de replicación intrasujeto o Diseño Experimental de Caso Único (DECU) (Barlow \& Hersen, 1984; Cazabat, 2013; Richaud de Minzi, 2008). 
Los DECU deben ser convenientemente diferenciados de los estudios de caso, los cuales son estudios post hoc. Los estudios con DECU pretenden controlar las fuentes de amenaza a la validez externa e interna, por medio de medidas repetidas, como se verá más adelante (Barker et al., 2002; Mc Millan \& Morley 2010; Turpin, 2005). Los criterios enunciados por el grupo de tareas de la American Psychological Association sobre validez de los tratamientos, permiten concluir que tanto los DECU como las pruebas aleatorizadas controladas proveen buen control de la validez interna y permiten inferir relaciones causales sobre la eficacia del tratamiento, como para establecer la validez de un tratamiento (Chambless et al., 1998).

Los DECU vinculan la actividad científica con la práctica clínica. Esto permite la integración de métodos formales de investigación con la práctica diaria, permitiendo abordar lo particular de lo individual y su complejidad (Barker et al., 2002). Esto difícilmente se pueda lograr con diseños de grupo. En los DECU, cada individuo sirve como su propio control. Esto se logra por medio de mediciones repetidas, que permiten monitorear minuciosamente cualquier cambio (Barker et al., 2002).

Los criterios para aplicar este tipo de diseño son los siguientes: (a) el paciente debe presentar un problema relativamente crónico y relativamente estable, como para que cualquier variación pueda ser atribuida sin dudas al tratamiento instituido, (b) el tratamiento debe tener efectos más o menos inmediatos, así como también su retirada debe tener efectos más o menos inmediatos y (c) el resultado esperado debe ser claro, objetivo y mensurable (Beins \& McCarthy, 2012).

El propósito de la línea de base es proveer un punto de comparación, por lo que debe ser tan extensa como sea necesario para que aparezca un patrón estable, de tal manera que se pueda identificar un cambio luego del establecimiento del tratamiento o la intervención (McMillan \& Morley, 2010). Por lo general, una línea de base de 3 semanas suele ser suficiente (Barlow \& Hersen, 1984; McMillan \& Morley, 2010).
Se estableció primeramente una línea de base de la sintomatología presentada, a lo largo de tres semanas, administrando la DTS y el OQ-45 con una frecuencia semanal (Charney et al., 1998; Lambert et al., 2008). A la finalización de esta fase, los participantes fueron asignados aleatoriamente a tres condiciones experimentales:

1.- la aplicación del protocolo EMDR durante tres sesiones, sin ningún tipo de estimulación, con auriculares en silencio (tratamiento placebo 1),

2.- la aplicación del protocolo EMDR durante tres sesiones, con estimulación bilateral auditiva simultánea (no alternada) (tratamiento placebo 2) y

3.- la aplicación del protocolo estándar de EMDR, con Estimulación Auditiva Bilateral Alternada, durante tres sesiones (tratamiento activo).

A partir de la cuarta sesión se les anunció que recibirían estimulación bilateral alternadamente, completando así el tratamiento.

El diseño utilizado fue de doble ciego (ni el terapeuta ni el paciente sabían a cuál de las tres condiciones había sido asignado cada paciente). La estimulación suministrada por medio de auriculares hizo que esto fuera posible, por lo que el terapeuta no tuvo conocimiento de qué tipo de estimulación estaba recibiendo cada participante. A partir de la cuarta sesión se aplicó el tratamiento estándar de EMDR con Estimulación Auditiva Bilateral Alternada (tratamiento activo), hasta la finalización del tratamiento, ya sea por haber llegado a la décima sesión, o por haber llegado a una resolución sintomática antes de las 10 sesiones (ver Cuadro 1).

\section{Resultados}

Tal como fue diseñado, cada participante, luego de establecerse la línea de base de tres semanas, recibió aleatoriamente, una de las tres condiciones experimentales durante tres 
sesiones. En la cuarta sesión de tratamiento se les anunció que iban a recibir una Estimulación Auditiva Bilateral Alternada, aplicando de tal manera el protocolo estándar de EMDR hasta la finalización del tratamiento. Durante la segunda fase de la aplicación de las condiciones experimentales, el terapeuta nunca estuvo al tanto del tipo de estimulación recibida por el paciente, manteniéndose de esta manera la condición de doble ciego.

Detalladamente, se pudo diseñar un procedimiento placebo, es decir, sin el componente supuestamente activo de EMDR (la Estimulación Auditiva Bilateral Alternada). El terapeuta se mantuvo sin conocer el tipo de estimulación utilizada hasta el final del estudio. Asimismo, los participantes aceptaron el procedimiento terapéutico integralmente, sin poner en duda el tipo de estimulación utilizada. Es decir que los procedimientos empleados resultaron creíbles para ambos, tanto para los pacientes como para el terapeuta.

No se analizarán los resultados de las mediciones realizadas con la DTS y el OQ45.2 , porque no es el objetivo del estudio que se informa ${ }^{1}$.

\section{DISCUSIÓN}

Como queda dicho, Borkovec y Sibrava (2005) plantean tres objeciones al uso de diseños a doble ciego en psicoterapia: (a) la primera es de orden conceptual: si se administra un tratamiento inerte y aun así ocurre algún cambio, éste se debe a algún tipo de mecanismo psicológico, por lo cual el pla-

\footnotetext{
No se informan dichos resultados porque sería necesario desarrollar temas tales como estudios de eficacia y efectividad, características psicométricas de los instrumentos utilizados, teoría y método de análisis gráfico de los resultados (dado que los DECU tienen una particular manera de analizar e interpretar sus resultados que requerirían una introducción teórica fundamentada). Por otra parte, la falta de exposición de estos resultados no afecta a la consecución de los objetivos planteados inicialmente.
}

cebo no era inerte dado que afirman "por lo tanto, metodológicamente, es imposible crear un procedimiento psicológico de control que sea inerte" (p. 807), (b) los autores asumen que el terapeuta / investigador necesariamente estará al tanto de estar administrando un tratamiento placebo, contaminando con sus expectativas los resultados, y es prácticamente imposible crear un procedimiento placebo con el mismo grado de credibilidad que el tratamiento activo y (c) plantean el problema ético de mantener en un tratamiento inerte a un paciente por un plazo determinado, que podría ocasionar un daño.

En respuesta a la objeción (a), se puede contestar que es un problema conceptual y por lo tanto depende de la definición de placebo que se adopte. Según la definición mencionada anteriormente de Shapiro y Morris (1978, ver Borkovec \& Sibrava 2005), placebo es

"cualquier terapia o componente de terapia que es usado deliberadamente por su efecto psicológico o psicofisiológico no específico, o que es usado por su efecto presupuesto, pero que no tiene actividad específica para la condición que está siendo tratada" (p. 807).

La psicoterapia EMDR asume que la estimulación bilateral alternada es el principal ingrediente activo del tratamiento, por lo que suministrar el protocolo de EMDR sin la Estimulación Auditiva Bilateral Alternada (condiciones de estimulación simultánea y silencio de este estudio) tendría un efecto no específico, respondiendo así a esta definición de placebo.

En cuanto a la objeción (b): en este estudio piloto se diseñó un procedimiento placebo igualmente creíble tanto para el paciente como para el terapeuta, ya que sólo difería del protocolo activo en la condición de estimulación aplicada, desconociendo los participantes cuál era el verdadero procedimiento. Y durante todo el estudio el terapeuta se mantuvo ciego a la condición aplicada en la fase correspondiente, ya que los 
participantes no hicieron comentario alguno sobre la estimulación recibida.

En cuanto a la objeción (c): por un lado, según los autores que discuten el papel jugado por la estimulación bilateral alternada, el protocolo de EMDR es similar a la terapia de exposición (Bergman, 2000; Lee et al., 2006; Spector, 2007). Según este punto de vista, al suministrar el placebo, en principio, se le estaría suministrando al participante un protocolo similar a la terapia de exposición, terapia que cuenta con suficiente evidencia empírica. Por otro lado, la sintomatología postraumática presentada por los pacientes tenía mucho tiempo de evolución, por lo que tres semanas sin tratamiento activo no proporcionaría un sufrimiento adicional significativo, ni tendría impacto sobre otras áreas de su vida que ya no hubiera tenido. Esto puede ser corroborado gracias a la información del monitoreo, sesión a sesión, realizado por medio de la aplicación de las escalas pertinentes para evaluar la evolución del estudio. Además, en caso de ser necesario, la realización de este monitoreo permitiría tomar la decisión de interrumpir el estudio si el participante experimentara mayor sufrimiento o un empeoramiento notorio de su sintomatología. En tal caso, podría suministrarse de inmediato uno de los tratamientos de probada eficacia, para mitigar su sufrimiento, salvaguardando así la integridad del paciente, y respetando el principio de primum non nocere.

Sin embargo, sin duda éste es un factor a considerar. El tiempo que todo estudio requiere para establecer la línea de base, en futuros estudios, debería ser evaluado en cada caso específico, determinando así la conveniencia de su aplicación. En tales casos debería ser ponderado tanto el tipo de trastorno sufrido como el tiempo de evolución del mismo, para la toma de decisión acerca de la inclusión en un estudio de este tipo.

Herbert y Gaudiano (2005) sugieren que con aquellas terapias conocidas como power therapies (terapias poderosas) sería posible diseñar procedimientos placebo a los que el terapeuta podría ser ciego. Dichas terapias son, entre otras (además de EMDR), TFT
(Thought Field Therapy - Terapia del Campo del Pensamiento), TIR (Traumatic Incident Reduction - Reducción de Incidente Traumático) y VKD (Visual Kinesthesic Dissociation - Disociación Viso-Kinestésica) (Carbonell \& Figley, 1999). Debido a que, al menos en algunas de sus versiones, estas terapias consisten en procedimientos estandarizados (tales como los descriptos, o estimulación por medio de digitopresión de diversos puntos de la acupuntura en el caso de TFT, por ejemplo) que son pasibles de ser ocultados al terapeuta, y a que sus resultados son relativamente inmediatos, es posible pensar en procedimientos placebo para cada uno de ellos, y por lo tanto en un diseño a doble ciego, para varios de los procedimientos mencionados, tal como se hizo en este estudio.

Una de las dificultades que se presenta, es que la aplicación del procedimiento placebo, tanto por razones técnicas como éticas, no puede prolongarse mucho. Pero esta dificultad puede ser superada gracias a la rapidez del efecto de estas terapias (entre una y cuatro horas de tratamiento, en promedio, según Carbonell y Figley, 1999) que no harían necesarios largos períodos de aplicación del placebo.

En principio este estudio tiene las limitaciones relativas al carácter de estudio piloto y una de ellas consiste en que el tratamiento ofrecido fue gratuito, mientras que diseños como los DECU tienen un enfoque naturalista, aspirando a ser lo más parecidos a la práctica real, de tal manera de aumentar su validez externa.

Otra limitación es que este estudio puso a prueba sólo la viabilidad del uso de placebos psicológicos y del diseño a doble ciego en estudios de resultados en psicoterapia, no la efectividad del EMDR o el papel que cumple la estimulación en el procesamiento de los recuerdos traumáticos, si es que cumple alguno.

En futuros estudios sería conveniente mejorar el diseño empleado, de tal manera que si en algún caso se revelara el tipo de estimulación recibida, esto no obligara a desechar a los demás participantes. 
La observación informal del autor apoya la idea de que los participantes encontraron la condición placebo como altamente creíble. Sin embargo, es necesario destacar que la credibilidad del placebo no fue formalmente evaluada.

Debe tenerse en cuenta que, dado que no existe un estándar para el análisis estadístico de los datos obtenidos mediante los DECU, sus resultados son difícilmente generalizables a poblaciones, y no son pasibles de ser incluidos en meta-análisis.

Una de las fortalezas más importantes es el carácter experimental de los DECU, que permite establecer relaciones causales. También que los DECU son diseños que no requieren de grandes cantidades de dinero ni un gran número de participantes, y pueden ser realizados por cualquier psicoterapeuta en el ámbito de su propio consultorio.

Las posibilidades que abren la aplicación de placebos psicológicos y los diseños a doble ciego en psicoterapia son múltiples y prometedoras.

Una posible investigación, a partir de la experiencia, sería tratar de registrar y analizar las apreciaciones y predicciones del terapeuta acerca de qué tipo de procedimiento está siendo aplicado (placebo o activo). A partir de la experiencia acumulada en cientos de sesiones de EMDR, el autor ha identificado cierto patrón de procesamiento de los recuerdos traumáticos, que como ya se manifestó, termina cuando se arriba a recuerdos emocionalmente cargados neutra o positivamente. En el caso de la aplicación de los procedimientos placebo, ese patrón de procesamiento no fue identificado, no arribándose al recuerdo neutro o positivo, de tal manera que el autor pudo predecir a partir de esos datos que se estaba aplicando el procedimiento placebo, confirmándose con posterioridad al momento de develar la condición experimental aplicada. De esta manera, mediante la sistematización del registro de las impresiones y predicciones del terapeuta, tanto a partir de la observación del proceso desarrollado en la sesión, como del informe por parte del paciente del proceso experimentado entre sesión y sesión, acerca del tipo de procedimiento aplicado, puede obtenerse un apoyo empírico importante para el papel desempeñado por la estimulación bilateral alternada. Esto también sería aplicable a otros procedimientos como los mencionados de las power therapies.

Según algunos autores (Carr, 2009; Freeman \& Power, 2007; Rubin, 2008) los factores inespecíficos de las diferentes psicoterapias darían cuenta de hasta el $66 \%$ de la variancia de los resultados positivos. Con este diseño y el empleo de placebos, también sería posible estudiar los efectos inespecíficos del contacto con el psicoterapeuta, al observar la evolución experimentada por aquellos sujetos que reciben el placebo. En particular con un DECU de tipo ABAB (es decir, línea de base, aplicación de la condición experimental, retirada de la misma y vuelta a aplicarla), podrían estudiarse los efectos inespecíficos de la relación terapéutica y cómo se sostienen en el tiempo.

El uso coordinado del placebo psicológico con los diversos tipos de DECU, abre múltiples posibilidades de combinaciones que pueden ser adaptadas a diversos objetivos y aplicadas a diversas formas de psicoterapia.

En la literatura revisada no se encontró referencia alguna a escalas $u$ otros instrumentos adecuados para llevar a cabo la evaluación de la credibilidad del placebo. Sería aconsejable establecer criterios para el desarrollo de placebos psicológicos creíbles, así como también desarrollar instrumentos para evaluar su credibilidad.

Si bien en este estudio presentado el diseño a doble ciego fue aplicado por medio de un Diseño Experimental de Caso Único, las características del procedimiento placebo lo hacen fácilmente adaptable a diseños de pruebas clínicas aleatorizadas (RCT) y a diseños de estudio de comparación entre grupos, que son hoy el estándar en investigación clínica. 
CUADRO 1

DISEÑO DEL ESTUDIO

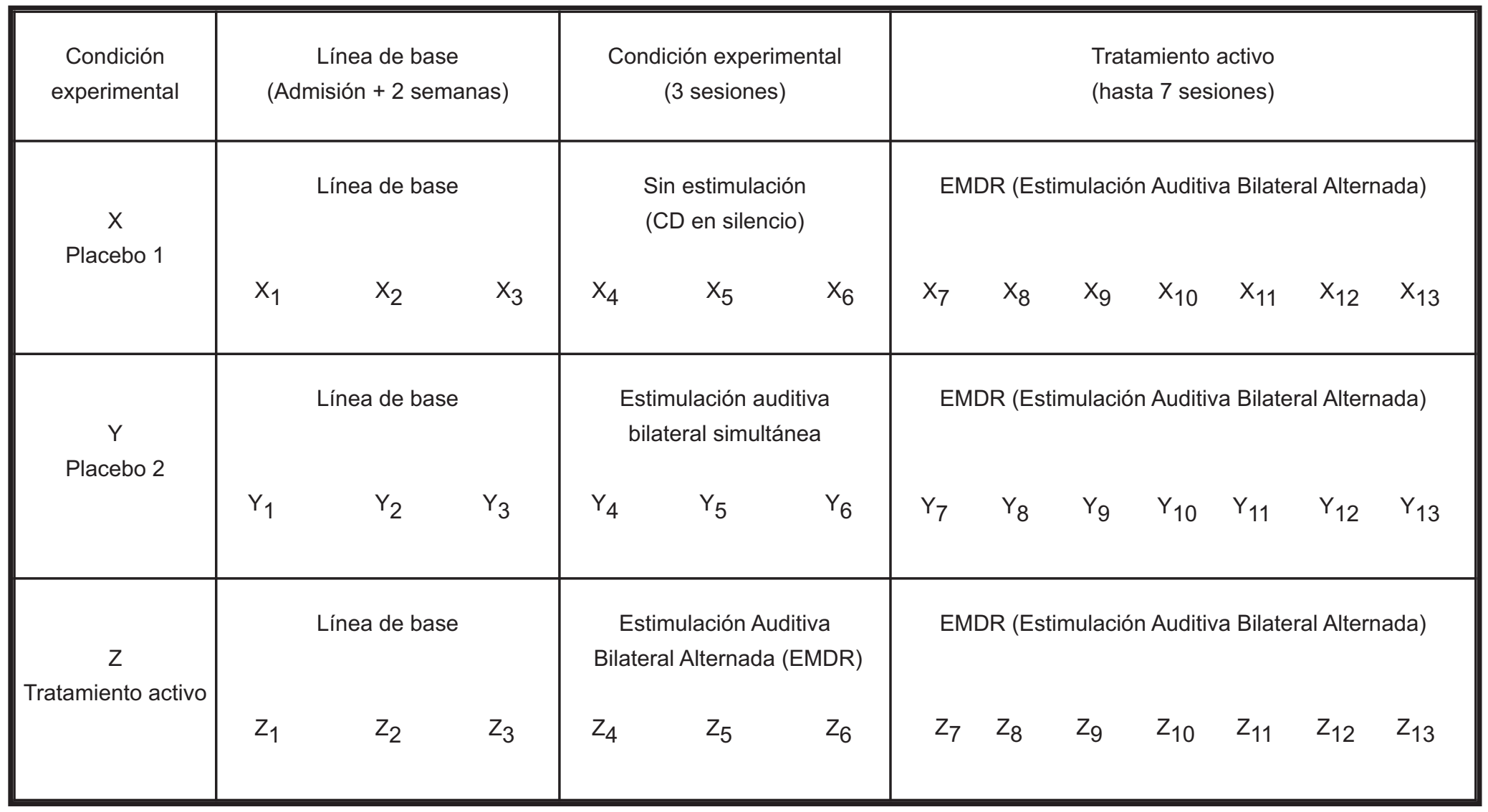




\section{REFERENCIAS BIBLIOGRÁFICAS}

Albright, D.L., Thyer, B., Becker, B. \& Rubin, A. (2011). Eye Movement Desensitization and Reprocessing (EMDR) for Posttraumatic Stress Disorder (PTSD) in combat veterans. Campbell Systematic Reviews - PROTOCOL. Recuperado el 15 de julio de 2013 de http://www. campbellcollaboration.org/lib/down load/16 25/\&sa $=$ U\&ei $=0 A M \quad 7$ Upq NL6jx2AAXAz ICoC\&ved $=0$ CAcQFjAA\&client $=$ internal-uds uds-cse\&usg=AFQjCNH5kPqBDEgS O5cjC SdGH4yslPIw

Barker, C., Pistrang, N. \& Elliott, R. (2002). Research methods in clinical psychology: An introduction for students and practitioners (2da. ed.). West Sussex, UK: Wiley.

Barkham, M., Hardy, G.E. \& Mellor-Clark, J. (2010). Developing and delivering practicebased evidence. West Sussex, UK: Wiley.

Barlow, D. \& Hersen, M. (1984). Single case experimental designs. Strategies for studying behavior change (2da. ed.). New York: Pergamon.

Beins, B.C. \& McCarthy, M.A. (2012). Research methods and statistics. Boston: Pearson.

Bergman, U. (2000). Further thoughts on the neurobiology of EMDR: The role of the cerebellum in accelerated information processing. Traumatology, 4(3), 175-200. http://dx.doi.org/ 10.1177/153476560000600303

Borkovek, T.D. \& Onken, L.S. (2002). Recommendations for research concerning the use of placebos in clinical trials to test behavioral interventions. En H.A. Guess, A. Kleinman, J.W. Kusek \& L.W. Engel (Eds.), The science of the placebo toward an interdisciplinary research agenda (pp. 306-310). London: BMJ Books.

Borkovec, T.D. \& Sibrava N.J. (2005). Problems with the use of placebo conditions in psychotherapy research, suggested alternatives, and some strategies for the pursuit of the placebo phenomenon. Journal of Clinical Psychology,
61(7), 805-818. http://dx.doi.org/10.10 02/jclp. 20127

Carbonell, J \& Figley, C. (1999). A systematic clinical demonstration of promising PTSD treatment approaches. Traumatology, 5(1), 32-48. http://dx.doi.org/10.1177/15347656 9900500106

Carr, A. (2009). What works with children, adolescents, and adults?: A review of research on the effectiveness of psychotherapy. NY: Routledge.

Cazabat, E.H. (2013). De clínico a investigador: La aplicación de diseños experimentales de caso único al contexto clínico [From clinician to researcher: The application of single case experimental designs to the clinical context]. Revista Argentina de Clínica Psicológica, 22 (3), 239-248.

Chambless, D.L., Baker, M.J., Baucom, D.H., Beutler, L.E., Calhoun, K.S., Crits-Christoph, P. et al. (1998). Update on empirically validated therapies, II. The Clinical Psychologist, 51(1), 3-16.

Chambless, D. \& Ollendick, T. (2001). Empirically supported psychological interventions: Controversies and evidence. Annual Review of Psychology, 52, 685-716.

Chambless, D., Sanderson, W., Shoham, V., Johnson, S., Pope, K., Crits-Shristoph, P. et al. (1996). An update on empirically validated therapies. The Clinical Psychologist, 49(2), 518.

Charney, D., Davidson, J., Friedman, M. Judge, R., Keane, T., McFarlane, S. et al. (1998). A consensus meeting on effective research practice in PTSD. CNS Spectrums, 3(7), (Suppl. 2), 7-10.

Christman S.D., Garvey, K.J., Propper R.E. \& Phaneuf, K.A. (2003). Bilateral eye movements enhance the retrieval of episodic memories. Neuropsychology, 17(2), 221-229. http://dx. doi.org/10.1037/0894-4105.17.2.221

Cusack, K. \& Spates, C.R. (1999). The cognitive dismantling of eye movement desensitization 
and reprocessing (EMDR) treatment of posttraumatic stress disorder (PTSD). Journal of Anxiety Disorders, 13(1), 87-99. http://dx. doi.org/10.1016/S0887-6185(98)00041-3

Davidson, J.R.T., Book, S.W., Colket, J.T., Tupler, L.A., Roth, S., David, D. et al. (1997). Assessment of a new self-rating scale for posttraumatic stress disorder. Psychological Medicine, 27, 153-160. http://dx.doi.org/10.1017/S0 033291796004229

Davidson, P.R. \& Parker, K.C.H. (2001). Eye Movement Desensitization and Reprocessing (EMDR): A meta-analysis. Journal of Consulting and Clinical Psychology, 69(2), 305-316. http://dx.doi.org/10.1037/0022-006X.69.2.305

Devilly, G.J. (2005). Power therapies and possible threats to the science of psychology and psychiatry. Australian and New Zealand Journal of Psychiatry, 39(6), 437-445. http://dx.doi. org/10.1111/j.1440-1614.2005.01601.x

Ehlers, A., Bisson, J., Clark, D.M., Creamer, M., Pilling, S., Richards, D. et al. (2010). Do all psychological treatments really work the same in posttraumatic stress disorder? Clinical Psychological Review, 30(2), 269-276. http://dx. doi.org/10.1016/j.cpr.2009.12.001

Fernández Álvarez, H., Hirsch, H., Maristany, M. \& Torrente, F. (2005). Propiedades psicométricas del OQ-45.2 en la Argentina: Un estudio piloto [OQ-45.2 psychometric properties in Argentina: A pilot study]. Trabajo presentado en el Congreso Mundial de Psicoterapias. Buenos Aires, Argentina.

Freeman, C. \& Power, M. (2007). Introduction. En C. Freeman \& M. Power (Eds.), Handbook of evidence-based psychotherapies: A guide for research and practice (pp. 3-14). West Sussex, UK: Wiley.

Goodwin, C.J. (2010). Research in psychology. Methods and designs (6ta. ed.). New Jersey: Wiley.

Haworth, J. (Ed.) (1996). Psychological research. Innovative methods and strategies. London: Routledge.
Heppner, P.P., Wampold, B.E. \& Kivlighan Jr., D.M. (2008). Research design in counseling (3ra. ed.). Belmont, CA: Thomson.

Herbert, J.D. (2003). The science and practice of empirically supported treatments. Behavior. Modification, 27, 412-430. http://dx.doi.org/ 10.1177/0145445503027003008

Herbert, J.D. \& Gaudiano, B.A. (2005). Introduction to the special issue on the placebo concept in psychotherapy. Journal of Clinical Psychology, 61(7), 787-790. http://dx.doi.org/10.1002/ jclp. 20125

Herbert, J.D. \& Sageman, M. (2004). "First Do No Harm": Emerging guidelines for the treatment of posttraumatic reactions. En G. Rosen (Ed.), Posttraumatic stress disorder: Issues and controversies. West Sussex, UK: Wiley. http://dx.doi.org/10.1002/9780470713570.ch 11

Howitt, D. \& Cramer, D. (2011). Introduction to research methods in psychology (3ra. ed.). Harlow (England): Prentice Hall.

Krauth, J. (2000). Experimental design. A handbook and dictionary for medical and behavioral research. Amsterdam: Elsevier.

Lambert, M.J. \& Goates, M.K. (2002). Efficacy. En M. Hersen \& W. Sledge (Eds.), Encyclopedia of Psychotherapy (pp. 715-718). NY: Elsevier.

Lambert, M., Hansen, N. \& Bauer, S. (2008). Assessing the clinical significance of outcome results. En A. Nezu \& C. Maguth Nezu (Ed.), Evidence-based outcome research: A practical guide to conducting randomized controlled trials for psychosocial interventions (pp. 359378). New York: Oxford University Press.

Lee, C.W., Taylor, G. \& Drummond, P.D. (2006). The active ingredient in EMDR: Is it traditional exposure or dual focus of attention? Clinical Psychology and Psychotherapy, 13, 97-107. http://dx.doi.org/10.1002/cpp.479

Lilienfeld, S.O., Ritschel, L.A., Lynn, S. J., Cautin, R.L. \& Latzman, R.D. (2013). Why many clinical psychologists are resistant to evidence- 
based practice: Root causes and constructive remedies. Clinical Psychology Review, 33(7), 883-900. http://dx.doi.org/10.1016/ j.cpr. 2012. 09.008

Marczyk, G., DeMatteo, D. \& Festinger, D. (2005). Essentials of research design and methodology. New Jersey: Wiley.

Maristany M. \& Fernández Álvarez, H. (en prensa). Evaluación de resultados en psicoterapia: Estudio de las propiedades psicométricas del OQ-45.2, en Argentina [Outcomes assessment in psychotherapy. Psychometric properties study of OQ-45.2, in Argentina]. Revista Argentina de Clínica Psicológica.

Maxfield, L. (2007). Current status and future directions for EMDR Research. Journal of EMDR Practice and Research, 1(1), 6-14. http://dx.doi.org/10.1891/1933-3196.1.1.6

McLeod, J. (2003). Doing counselling research (2da. ed.). London: SAGE.

McMillan, D. \& Morley, S. (2010). Single case quantitative methods for practice-based evidence. En M. Barkham, G.E. Hardy \& J. Mellor-Clark (Eds.), Developing and delivering practice-based evidence: A guide for the psychological therapies (pp. 109-138). West Sussex, UK: Wiley. http://dx.doi.org/10.1002/ 9780470687994.ch5

McNally, R.J. (1999). Research on Eye Movement Desensitization and Reprocessing (EMDR) as a treatment for PTSD. PTSD Research Quarterly, 10(1), 1-3.

Nowill, J. (2010). A critical review of the controversy surrounding eye movement desensitization and reprocessing. Counselling Psychology Review, 25(1), 63-70.

Oren, E. \& Solomon, R. (2012). EMDR therapy: An overview of its development and mechanisms of action. Revue Européenne de Psychologie Appliquée, 62, 197-203. http://dx.doi. org/10.1016/j.erap.2012.08.005

Propper, R.E. \& Christman, S.D. (2008). Interhemispheric interaction and saccadic horizontal eye movements implications for episodic memory, EMDR, and PTSD. Journal of EM DR Practice and Research, 2(4), 269-281. http://dx.doi.org/10.1891/1933-3196.2.4.269

Richaud de Minzi, M.C. (2008). Cuaderno de diseños de replicación intrasujeto (Diseño de caso único) [Intrasubject replication designs handout (Single case design)]. Manuscrito no publicado.

Roberts, M.C. \& Ilardi, S.S. (2005). Research methodology and clinical psychology: An overview. En M.C. Roberts \& S.S. Ilardi (Eds.), Handbook of research methods in clinical psychology (pp. 3-12). Oxford, UK: Blackwell.

Rothbaum, B.O. \& Davis, M. (2004). Aplicación de los principios del aprendizaje para el tratamiento de reacciones postraumáticas [Applying learning prtinciples to the treatment of post trauma reactions]. Revista de Psicotrauma para Iberoamérica, 3(2), 4-13.

Rubin, A. (2003). Unanswered questions about the empirical support for EMDR in the treatment of PTSD: A review of research. Traumatology, 9(1), 4-30. http://dx.doi. org/10.1177/15347 6560300900102

Rubin, A. (2008). Practitioner's guide to using research for evidence-based practice. New Jersey: Wiley.

Santibáñez Fernández, P.M., Román Mella, M.F. \& Vinet, E.V. (2009). Efectividad de la psicoterapia y su relación con la alianza terapéutica [Effectiveness of psychotherapy and its relation to the therapeutic alliance]. Interdisciplinaria, 26(2), 267-287.

Schubert, S. \& Lee, C.W. (2009). Adult PTSD and its treatment with EMDR: A review of controversies, evidence, and theoretical knowledge. Journal of EMDR Practice and Research, 3(3), 117-132. http://dx.doi.org/10 1891/1933-319 6.3.3.117

Servan-Schreiber, D., Schooler, J., Dew, M.A., Carter, C. \& Bartone, P. (2006). Eye movement desensitization and reprocessing for Posttraumatic Stress Disorder: A pilot blinded, ran- 
domized study of stimulation type. Psychotherapy and Psychosomatics, 75, 290-297. http://dx.doi.org/10.1159/000093950

Shapiro, F. (1995). Eye movement desensitization and reprocessing: Basic principles, protocols, and procedures. New York: Guilford.

Shapiro, F. (2001). Eye movement desensitization and reprocessing: Basic principles, protocols, and procedures (2da. ed.). New York: Guilford.

Shapiro, F. (2007). EMDR, adaptive information processing, and case conceptualization. Journal of EMDR Practice and Research, 1(2), 68-87. http://dx.doi.org/1 0.1016/10.1891/19 33-3196.1.2.68

Shapiro, F. (2012). EMDR therapy: An overview of current and future research. Revue Européenne de Psychologie Appliquée, 62, 193-195. http://dx.doi.org/10.1016/j.erap.2012.09.005

Simons, A.D. \&. Wildes, J.E (2005). Therapy and interventions research with adults. En M.C. Roberts \& S.S. Ilardi (Eds.), Handbook of research methods in clinical psychology (pp. 329-351). Oxford, UK: Blackwell Publishing.

Spector, J. (2007). Eye Movement Desensitization and Reprocessing (EMDR). En C. Freeman \& M. Power (Eds.), Handbook of evidence-based psychotherapies. A guide for research and practice (pp. 93-109). Chichester: Wiley.

Stickgold, R. (2002). EMDR: A putative neurobiological mechanism of action. Journal of
Clinical Psychology, 58(1), 61-75. http://dx. doi.org/10.1002/jclp.1129

Turpin, G. (2005). Single case methodology and psychotherapy evaluation: From research to practice. En C. Mace, S. Moorey \& B. Roberts (Eds.), Evidence in the psychological therapies: A critical guide for practitioners (pp. 90-110). Taylor \& Francis e-Library.

Villafañe, A., Milanesio, M.S., Marcellino, C.M. \& Amodei, C. (2003). La evaluación del trastorno por estrés postraumático: Aproximación a las propiedades psicométricas de la Escala de Trauma de Davidson [The assessment of posttraumatic stress disorder: Davidson Trauma Scales psychometric properties approach]. Evaluar, 3, 80-93.

von Bergen, A. \& de la Parra, G. (2002). OQ-45, Cuestionario para la Evaluación de Resultados y Evolución en Psicoterapia: Adaptación, validación e indicaciones para su aplicación e interpretación [OQ-45, an Outcome Questionnaire for monitoring change in psychotherapy: Adaptation, validation and indications for its application and interpretation]. Revista Terapia Psicológica, 20(2), 161-175.

Wojciechowski, F.L. (1984). Double blind research in psychotherapy. Lisse, Netherlands: Swets \& Zeitlinger.
Práctica Privada Ciudad Autónoma de Buenos Aires República Argentina

Fecha de recepción: 21 de septiembre de 2013 Fecha de aceptación: 16 de enero de 2014 
